\title{
EFFECTIVE EQUIDISTRIBUTION OF THE REAL PART OF ORBITS ON HYPERBOLIC SURFACES
}

\author{
JIMI L. TRUELSEN
}

(Communicated by Kathrin Bringmann)

\begin{abstract}
For non-cocompact Fuchsian groups with finite covolume we prove that the real part of the orbit of a point in the upper half-plane is equidistributed with an effective error term. This extends previous results by A. Good, M. Risager, and Z. Rudnick. We use the equidistribution result to generalize a theorem by F. Chamizo.
\end{abstract}

\section{INTRODUCTION}

It is well known that the group $\mathrm{SL}_{2}(\mathbf{R})$ acts on the upper half-plane $\mathbf{H} \subset \mathbf{C}$ as Möbius transformations. Let $\Gamma \subset \mathrm{SL}_{2}(\mathbf{R})$ be a Fuchsian group with finite covolume and let $d(\cdot, \cdot)$ denote the hyperbolic distance on $\mathbf{H} \cong \mathbf{R} \times \mathbf{R}_{+}$derived from the Poincaré metric $d s^{2}=y^{-2}\left(d x^{2}+d y^{2}\right)$. With this metric elements in $\mathrm{SL}_{2}(\mathbf{R})$ act as orientation-preserving isometries on $\mathbf{H}$. It has been proved by numerous authors ([2], 4], [5], 6], 7], 9], 10]) that there exists $a<1$ such that for $z_{1}, z_{2}$ fixed and $R>0$,

$$
\#\left\{\gamma \in \Gamma: d\left(\gamma z_{1}, z_{2}\right) \leq R\right\}=\frac{\mathfrak{K}_{\Gamma} \pi}{\operatorname{Vol}(\Gamma \backslash \mathbf{H})} e^{R}+O\left(e^{a R}\right)
$$

where

$$
\mathfrak{K}_{\Gamma}= \begin{cases}1 & \text { if }\left({ }^{-1}-1\right) \notin \Gamma, \\ 2 & \text { if }\left({ }^{-1}-1\right) \in \Gamma .\end{cases}
$$

This is known as the hyperbolic lattice point problem. In [8] a very general approach to lattice point counting in locally compact groups is presented.

In this paper we consider a variant of the hyperbolic lattice point problem. Throughout this paper we let $\Gamma$ denote a non-cocompact Fuchsian group of the first kind and we will assume that $\infty$ is a cusp of $\Gamma$ of width 1 . The last condition is not very strict, as we can always bring ourselves into this situation by conjugation. We let $\Gamma_{\infty}$ denote the stability group of the cusp $\infty$. If $\mathfrak{K}_{\Gamma}=1$ the group $\Gamma_{\infty}$ is generated by $\left(\begin{array}{cc}1 & 1 \\ & 1\end{array}\right)$. If $\mathfrak{K}_{\Gamma}=2$ the group $\Gamma_{\infty}$ is generated by $\left(\begin{array}{ll}1 & 1 \\ & 1\end{array}\right)$ and $\left(\begin{array}{ll}-1 & \\ & -1\end{array}\right)$.

Received by the editors June 18, 2011 and, in revised form, July 9, 2011.

2010 Mathematics Subject Classification. Primary 11B05, 11J71; Secondary 11M36.

The author was funded by a stipend from The Danish Agency for Science, Technology and Innovation. 
Define

$$
N_{\Gamma}(T ; z)=\#\left\{\gamma \in \Gamma_{\infty} \backslash \Gamma: \operatorname{Im}(\gamma z) \geq T^{-1}\right\} .
$$

Our approach to investigating (1.2) is via spectral theory of the automorphic Laplacian on $\Gamma \backslash \mathbf{H}$. We let $\Delta$ denote the automorphic Laplacian which is the selfadjoint closure of the operator

$$
-y^{2}\left(\frac{\partial^{2}}{\partial x^{2}}+\frac{\partial^{2}}{\partial y^{2}}\right)
$$

restricted to smooth functions on $\Gamma \backslash \mathbf{H}$ with compact support. It is well known that $\Delta$ has a continuous spectrum $[1 / 4, \infty$ ) (with multiplicity equal to the number of inequivalent cusps) as well as eigenvalues (counted with multiplicity)

$$
0=\lambda_{0}<\lambda_{1} \leq \lambda_{2} \leq \ldots,
$$

which are finite in number or tending to $\infty$. We write $\lambda_{j}=s_{j}\left(1-s_{j}\right)$, where $\sigma_{j}=\operatorname{Re}\left(s_{j}\right) \geq 1 / 2$. We remark that it is important for our applications that there is a spectral gap between the zeroth eigenvalue $\lambda_{0}=0$ and the first eigenvalue $\lambda_{1}$. In other words we have the strict inequality $\sigma_{1}<\sigma_{0}=1$.

A. Good [4] proved that for $z$ fixed and $T \geq 1$,

$$
N_{\Gamma}(T ; z)=\frac{T}{\operatorname{Vol}(\Gamma \backslash \mathbf{H})}+O\left(T^{\max \left(2 / 3, \sigma_{1}\right)}\right) .
$$

Here $\operatorname{Vol}(A)=\int_{A} y^{-2} d x d y$ denotes the hyperbolic volume on $\mathbf{H}$. The asymptotics in (1.3) is indeed the result we would expect since the hyperbolic volume of the infinite box

$$
\#\left\{x+i y \in \mathbf{H}: x \in[0,1], y \geq T^{-1}\right\}
$$

is $T$. In (2.8) below we obtain (1.3) with the error term $O\left(T^{\left(1+\sigma_{1}\right) / 2+\varepsilon}\right)$.

For a closed interval $I \subset \mathbf{R} / \mathbf{Z}$ we define

$$
N_{\Gamma}^{I}(T ; z)=\#\left\{\gamma \in \Gamma_{\infty} \backslash \Gamma: \operatorname{Im}(\gamma z) \geq T^{-1}, \operatorname{Re}(\gamma z) \in I\right\} .
$$

The main theorem of this paper is the following effective equidistribution result:

Theorem 1. Let $I \subset \mathbf{R} / \mathbf{Z}$ be a closed interval, $K \subset \Gamma \backslash \mathbf{H}$ a compact set, and $T \geq 1$. Then

$$
N_{\Gamma}^{I}(T ; z)=\frac{|I| T}{\operatorname{Vol}(\Gamma \backslash \mathbf{H})}+O\left(T^{\left(3+\sigma_{1}\right) / 4+\varepsilon}\right)
$$

uniformly for $z \in K$. The constant implied depends at most on $\Gamma$, $\varepsilon$, and $K$.

The asymptotics in the theorem was proved in [4] and [11] but without the error term. Our strategy is to combine the spectral theory arguments in 11 and $[12$.

Using the approximation argument in [12, Lemma 3.1] we can count lattice points in more general domains. We do not strive to obtain maximal generality on the domains, but we give a couple of examples. For $\Omega \subset \Gamma_{\infty} \backslash \mathbf{H}$ we define

$$
\mathcal{N}_{\Gamma}(\Omega ; z)=\#\left\{\gamma \in \Gamma_{\infty} \backslash \Gamma: \gamma z \in \Omega\right\} .
$$

When estimating $\mathcal{N}_{\Gamma}(\Omega ; z)$ we will view $\Omega$ as a subset of $[0,1] \times \mathbf{R}_{+}$rather than $\Gamma_{\infty} \backslash \mathbf{H}$. It will be clear as we go along that this does not make any difference. 
Let $[a, b] \subset[0,1]$ and $\phi:[a, b] \rightarrow \mathbf{R}_{+}$be bounded (here bounded means that $\overline{\phi([a, b])}$ is compact in $\mathbf{R}_{+}$) and integrable (thus $1 / \phi$ is integrable). We define

$$
G_{\phi}=\left\{x+i y \in \Gamma_{\infty} \backslash \mathbf{H}: a \leq x \leq b, y \geq \phi(x)\right\} .
$$

We easily check that

$$
\operatorname{Vol}\left(G_{\phi}\right)=\int_{a}^{b} \int_{\phi(x)}^{\infty} y^{-2} d y d x=\int_{a}^{b} \phi(x)^{-1} d x .
$$

The following theorem is natural and allows us to estimate the number of lattice points in rather general domains:

Theorem 2. Let $[a, b] \subset[0,1]$ and $\phi:[a, b] \rightarrow \mathbf{R}_{+}$be monotonic and bounded. Then

$$
\mathcal{N}_{\Gamma}\left(G_{\phi} ; z\right)=\frac{\operatorname{Vol}\left(G_{\phi}\right)}{\operatorname{Vol}(\Gamma \backslash \mathbf{H})}+O\left((1 / \inf \phi)^{\left(7+\sigma_{1}\right) / 8+\varepsilon}\right) .
$$

The constant implied depends at most on $\Gamma, \varepsilon$, and $z$.

The following is an immediate consequence of Theorem 2 ,

Corollary 3. Let $[a, b] \subset[0,1]$ and $f:[a, b] \rightarrow \mathbf{R}_{+}$be monotonic and bounded. Define $\phi_{T}(x)=f(x) / T$ for $x \in[a, b], T \geq 1$. Then

$$
\mathcal{N}_{\Gamma}\left(G_{\phi_{T}} ; z\right)=\frac{\operatorname{Vol}\left(G_{\phi_{T}}\right)}{\operatorname{Vol}(\Gamma \backslash \mathbf{H})}+O\left(T^{\left(7+\sigma_{1}\right) / 8+\varepsilon}\right) .
$$

The constant implied depends at most on $\Gamma, \varepsilon, z$, and inf $f$. Also we have

$$
\operatorname{Vol}\left(G_{\phi_{T}}\right)=T \int_{a}^{b} f(x)^{-1} d x
$$

Let $[\alpha, \beta] \subset \mathbf{R}_{+}$and $F:[\alpha, \beta] \rightarrow[0,1]$ be monotonic. We define:

$$
\Omega_{F, T}=\left\{x+i y \in \Gamma_{\infty} \backslash \mathbf{H}: y \in[\alpha / T, \beta / T], \min (F) \leq x \leq F(T y)\right\} .
$$

In [1] the following theorem was proved:

Theorem 4. Let $[\alpha, \beta] \subset \mathbf{R}_{+}$and $F:[\alpha, \beta] \rightarrow[0,1]$ be monotonic and continuously differentiable. For $T>2$,

$$
\mathcal{N}_{\mathrm{SL}_{2}(\mathbf{Z})}\left(\Omega_{F, T} ; i\right)=\frac{3 T}{\pi} \int_{\beta^{-1}}^{\alpha^{-1}}(F(1 / t)-\min (F)) d t+O\left(T^{7 / 8} \log T\right) .
$$

The constant implied depends only on $\alpha$.

Using the ideas in Theorem 2 and Corollary 3 we can extend Theorem 4 to any non-cocompact Fuchsian group of the first kind with a cusp at $\infty$ of width 1 :

Proposition 5. Let $[\alpha, \beta] \subset \mathbf{R}_{+}$and $F:[\alpha, \beta] \rightarrow[0,1]$ be monotonic and continuous. For $T \geq 1$ we have

$$
\mathcal{N}_{\Gamma}\left(\Omega_{F, T} ; z\right)=\frac{T}{\operatorname{Vol}(\Gamma \backslash \mathbf{H})} \int_{\beta^{-1}}^{\alpha^{-1}}(F(1 / t)-\min (F)) d t+O\left(T^{\left(7+\sigma_{1}\right) / 8+\varepsilon}\right) .
$$

The constant implied depends at most on $\Gamma, \varepsilon, z$, and $\alpha$. 
The error term in Proposition 5 is worse than the error term in Theorem 4 in the case of the modular group. However, it is important to stress that the asymptotics found in Proposition 5 is not an "arithmetic" feature; rather it is a general property of non-cocompact finite volume hyperbolic surfaces. From Proposition 5 we easily deduce:

Corollary 6. Let $\Omega \subset \mathbf{H}$ be a closed and connected set bounded by straight line segments and $m_{1}$ curves $\xi_{j}$ of the form $\xi_{j}(t)=\left(t, f_{j}(t)\right)$, where $f_{j}:\left[a_{j}, b_{j}\right] \rightarrow \mathbf{R}_{+}$ is monotonic. Assume $\Omega \subset\left[0, m_{2}\right] \times \mathbf{R}_{+}$and set

$$
\left\{\alpha_{0}, \ldots, \alpha_{m}\right\}=\left\{a_{1}, b_{1}, \ldots, a_{m_{1}}, b_{m_{1}}\right\} \cup\left\{0,1, \ldots, m_{2}-1, m_{2}\right\},
$$

where $\alpha_{j-1}<\alpha_{j}$ for $j=1, \ldots, m$. Define

$$
L_{j}=\left\{z \in \mathbf{H}: \operatorname{Re}(z) \in\left[\alpha_{j-1}, \alpha_{j}\right]\right\} .
$$

Then

$$
\begin{aligned}
& \#\{\gamma \in \Gamma: \gamma z \in \Omega\} \\
& \quad=\frac{\mathfrak{K}_{\Gamma} \operatorname{Vol}(\Omega)}{\operatorname{Vol}(\Gamma \backslash \mathbf{H})}+O\left(\sum_{j=1}^{m}\left(1 / \inf \left\{\operatorname{Im}(z): z \in \Omega \cap L_{j}\right\}\right)^{\left(7+\sigma_{1}\right) / 8+\varepsilon}\right),
\end{aligned}
$$

where the constant implied depends at most on $\Gamma, \varepsilon$, and $z$.

\section{EQUidistribution}

In this section we prove Theorem 1] Following [1] we define

$$
V_{m}(z, s)=\sum_{\Gamma_{\infty} \backslash \Gamma} \operatorname{Im}(\gamma z)^{s} e(m \operatorname{Re}(\gamma z)),
$$

which is absolutely convergent for $\operatorname{Re}(s)>1$. The function $V_{m}(z, s)$ is not square integrable on $\Gamma \backslash \mathbf{H}$, but as remarked in [11] the function

$$
W_{m}(z, s)=V_{m}(z, s)-h(y) y^{s} e(m x)
$$

is. Here $h$ is a smooth, increasing function such that $h(y)=0$ for $y<T$ and $h(y)=1$ for $y>T+1, T \geq 1$. The series $V_{m}(z, s)$ satisfies the equation

$$
(\Delta-s(1-s)) V_{m}(z, s)=(2 \pi m)^{2} V_{m}(z, s+2) \text {. }
$$

Moreover,

$$
(\Delta-s(1-s)) W_{m}(z, s)=(2 \pi m)^{2} W_{m}(z, s+2)-H_{m}(z, s),
$$

where

$$
H_{m}(z, s)=h^{\prime \prime}(y) y^{s+2} e(m x)+2 h^{\prime}(y) y^{s+1} e(m x) .
$$

Note that $H_{m}(z, s)$ is compactly supported for $z \in \Gamma \backslash \mathbf{H}$. From [11, Proposition A.1] we know that $V_{m}(z, s)$ and $W_{m}(z, s)$ admit meromorphic continuations to $\operatorname{Re}(s)>$ $1 / 2$. The potential poles are at $s=\sigma_{j}$, where $\lambda_{j}=\sigma_{j}\left(1-\sigma_{j}\right)$ are the small eigenvalues of $\Delta$ (i.e. the eigenvalues smaller than $1 / 4$ ). For $m \neq 0, V_{m}(z, s)$ and $W_{m}(z, s)$ are regular at $s=1$. For $m=0$ we have

$$
\operatorname{res}_{s=1} W_{0}(z, s)=\operatorname{res}_{s=1} V_{0}(z, s)+O(1 / T)=1 / \operatorname{Vol}(\Gamma \backslash \mathbf{H})+O(1 / T) .
$$

The constant implied is absolute. 
We need the following estimates on $W_{m}(z, s)$ :

Lemma 7. Let $s=\sigma+i$ and $K \subset F$ be a fixed compact set. For $|t|>1$ and $\sigma>1 / 2+\kappa, \kappa>0$ we have

$$
W_{m}(z, s)=O\left(\left(m^{2}+1\right)|t|\right) .
$$

For $|t| \leq 1$ and $\sigma \in\left(\sigma_{1}+\kappa, 1-\kappa\right), \kappa>0$ we have

$$
W_{m}(z, s)=O\left(m^{2}+1\right) .
$$

The constants implied may depend on $K, \Gamma$, and $\kappa$.

Proof. The proof follows the proof of Lemma 2.1 in [12] closely. Assume first that $|t|>1$. We have

$$
\|R(s)\| \leq \frac{1}{\operatorname{dist}(s(1-s), \operatorname{spec}(\Delta))} \leq \frac{1}{|t|(2 \sigma-1)},
$$

where $\|\cdot\|$ denotes the operator norm and $R(s)=(\Delta-s(1-s))^{-1}$ is the resolvent. We see that

$$
\left\|W_{m}(z, s)\right\|_{2} \leq\left\|W_{0}(z, 3 / 2)\right\|_{2}
$$

for $\sigma \geq 3 / 2$. By (2.1) we obtain the $L^{2}$-bound

$$
\begin{aligned}
\left\|W_{m}(z, s)\right\|_{2} & \leq\|R(s)\|\left((2 \pi m)^{2}\left\|W_{m}(z, s+2)\right\|_{2}+\left\|H_{m}(z, s)\right\|_{2}\right) \\
& =O\left(\left(m^{2}+1\right) /|t|\right)
\end{aligned}
$$

for $\operatorname{Re}(s)>1 / 2+\kappa$. From (2.1) we also get

$$
\begin{aligned}
\| \Delta W_{m} & (z, s) \|_{2} \\
& \leq(2 \pi m)^{2}\left\|W_{m}(z, s+2)\right\|_{2}+\left\|H_{m}(z, s)\right\|_{2}+\left\|s(1-s) W_{m}(z, s)\right\|_{2} \\
& =O\left(\left(m^{2}+1\right)|t|\right),
\end{aligned}
$$

for $\operatorname{Re}(s)>1 / 2+\kappa$.

To obtain the desired pointwise bound (in fact we get a uniform bound) we use the same strategy as in [12, Lemma 2.1], which uses the Sobolev imbedding theorem and elliptic regularity theory. We omit the details. This leads to the bound

$$
\sup _{z \in K}\left|W_{m}(z, s)\right|=O\left(\left\|W_{m}(z, s)\right\|_{2}+\left\|\Delta W_{m}(z, s)\right\|_{2}\right)=O\left(\left(m^{2}+1\right)|t|\right) .
$$

Still the constants implied depend at most on $K, \Gamma$, and $\kappa$. This proves the case where $|t|>1$. For $|t| \leq 1$ and $\sigma \in\left(\sigma_{1}+\kappa, 1-\kappa\right)$ we have the bound

$$
\|R(s)\| \leq \sup _{\substack{|t| \leq 1 \\ \sigma \in\left(\sigma_{1}+\kappa, 1-\kappa\right)}} \max \left(\left|\lambda_{1}-(s(1-s))\right|^{-1},|1-(s(1-s))|^{-1}\right),
$$

which comes from the first inequality in (2.2). Note that this is finite since $\left[\sigma_{1}+\right.$ $\kappa, 1-\kappa] \times[-1,1]$ is compact. With this estimate we can proceed as before to obtain the desired bound on $W_{m}(z, s)$.

For $U>U_{0} \geq 1$ let $\psi_{U}: \mathbf{R}_{+} \rightarrow \mathbf{R}$ be a family of smooth decreasing functions such that

$$
\psi_{U}(t)= \begin{cases}1 & \text { if } t \leq 1-1 / U \\ 0 & \text { if } t \geq 1+1 / U\end{cases}
$$


and $\psi_{U}^{(j)}(t)=O\left(U^{j}\right)$ as $U \rightarrow \infty$. For $\operatorname{Re}(s)>0$ we let

$$
\Psi_{U}(s)=\int_{0}^{\infty} \psi_{U}(y) y^{s-1} d y
$$

denote the Mellin transform of $\psi_{U}$. Mean value estimates give us

$$
\Psi_{U}(s)=s^{-1}+O\left(U^{-1}\right) \quad \text { as } U \rightarrow \infty
$$

and for any $c>0$,

$$
\Psi_{U}(s)=O\left(\frac{1}{|s|}\left(\frac{U}{1+|s|}\right)^{c}\right) \quad \text { as }|s| \rightarrow \infty .
$$

The estimates are uniform for $\operatorname{Re}(s)$ bounded. By the Mellin inversion formula we get

$$
\begin{aligned}
\sum_{\gamma \in \Gamma_{\infty} \backslash \Gamma} e(m \operatorname{Re}(\gamma z)) & (1-h(\operatorname{Im}(\gamma z))) \psi_{U}\left(\operatorname{Im}(\gamma z)^{-1} T^{-1}\right) \\
= & \frac{1}{2 \pi i} \int_{(2)} W_{m}(z, s) \Psi_{U}(s) T^{s} d s
\end{aligned}
$$

Note that Lemma 7 ensures that the integral on the right-hand side is convergent. Moving the line of integration to the line $\operatorname{Re}(s)=\sigma_{1}+\varepsilon$ we get

$$
\begin{aligned}
& \frac{1}{2 \pi i} \int_{(2)} W_{m}(z, s) \Psi_{U}(s) T^{s} d s \\
& \quad=\operatorname{res}_{s=1}\left(W_{m}(z, s) \Psi_{U}(s) T^{s}\right)+\frac{1}{2 \pi i} \int_{\left(\sigma_{1}+\varepsilon\right)} W_{m}(z, s) \Psi_{U}(s) T^{s} d s \\
& \quad=\delta_{m, 0}(T / \operatorname{Vol}(\Gamma \backslash \mathbf{H})+O(T / U+1))+\frac{1}{2 \pi i} \int_{\left(\sigma_{1}+\varepsilon\right)} W_{m}(z, s) \Psi_{U}(s) T^{s} d s .
\end{aligned}
$$

We need to estimate the last integral. Applying Lemma 7 and (2.5) with $c=1+\varepsilon$ the part of the line integral with $|t|>1$ is

$$
O\left(\left(m^{2}+1\right) T^{\sigma_{1}+\varepsilon} U^{1+\varepsilon}\right) .
$$

The part of the line integral with $|t| \leq 1$ is estimated using Lemma 7 and (2.4), and we obtain

$$
O\left(\left(m^{2}+1\right) T^{\sigma_{1}+\varepsilon}\right)
$$

Consequently,

$$
\begin{aligned}
\sum_{\gamma \in \Gamma_{\infty} \backslash \Gamma} e(m \operatorname{Re}(\gamma z)) & (1-h(\operatorname{Im}(\gamma z))) \psi_{U}\left(\operatorname{Im}(\gamma z)^{-1} T^{-1}\right) \\
= & \delta_{m, 0}(T / \operatorname{Vol}(\Gamma \backslash \mathbf{H})+O(T / U))+O\left(\left(m^{2}+1\right) T^{\sigma_{1}+\varepsilon} U^{1+\varepsilon}\right) .
\end{aligned}
$$

Clearly,

$$
\sum_{\gamma \in \Gamma_{\infty} \backslash \Gamma} e(m \operatorname{Re}(\gamma z)) h(\operatorname{Im}(\gamma z)) \psi_{U}\left(\operatorname{Im}(\gamma z)^{-1} T^{-1}\right)=O(1)
$$

Let $\psi_{U}^{-}, \psi_{U}^{+}: \mathbf{R}_{+} \rightarrow \mathbf{R}$ satisfy (2.3) and

$$
\psi_{U}^{-} \leq \mathbf{1}_{(0,1]} \leq \psi_{U}^{+},
$$


where $\mathbf{1}_{(0,1]}$ denotes the characteristic function on $(0,1]$. We see that

$$
\sum_{\gamma \in \Gamma_{\infty} \backslash \Gamma} \psi_{U}^{-}\left(\operatorname{Im}(\gamma z)^{-1} T^{-1}\right) \leq N_{\Gamma}(T ; z) \leq \sum_{\gamma \in \Gamma_{\infty} \backslash \Gamma} \psi_{U}^{+}\left(\operatorname{Im}(\gamma z)^{-1} T^{-1}\right) .
$$

Thus (2.6) and (2.7) with $m=0$ and $U=T^{\left(1-\sigma_{1}\right) / 2}$ imply

$$
N_{\Gamma}(T ; z)=\frac{T}{\operatorname{Vol}(\Gamma \backslash \mathbf{H})}+O\left(T^{\left(1+\sigma_{1}\right) / 2+\varepsilon}\right) .
$$

We remark that (1.3) could be used in the estimate below, but it does not give rise to a better error term in the final result. Using (2.8) we see that

$$
\begin{aligned}
\sum_{\gamma \in \Gamma_{\infty} \backslash \Gamma} e(m \operatorname{Re}(\gamma z)) \psi_{U}^{+}\left(\operatorname{Im}(\gamma z)^{-1} T^{-1}\right) & -\sum_{\substack{\gamma \in \Gamma_{\infty} \backslash \Gamma \\
\operatorname{Im}(\gamma z) \geq T^{-1}}} e(m \operatorname{Re}(\gamma z)) \\
& =O\left(N_{\Gamma}\left(T\left(1+U^{-1}\right) ; z\right)-N_{\Gamma}(T ; z)\right) \\
& =O\left(T / U+T^{\left(1+\sigma_{1}\right) / 2+\varepsilon}\right) .
\end{aligned}
$$

This together with (2.6) and (2.7) gives

$$
\sum_{\substack{\gamma \in \Gamma_{\infty} \backslash \Gamma \\ \operatorname{Im}(\gamma z) \geq T^{-1}}} e(m \operatorname{Re}(\gamma z))=O\left(T / U+T^{\left(1+\sigma_{1}\right) / 2+\varepsilon}+m^{2} T^{\sigma_{1}+\varepsilon} U^{1+\varepsilon}\right)
$$

for $m \neq 0$. Let $I \subset \mathbf{R} / \mathbf{Z}$ be an interval. The Erdös-Turán inequality [3, Theorem III] implies that

$$
\frac{N_{\Gamma}^{I}(T ; z)}{N_{\Gamma}(T ; z)}=|I|+O\left(M^{-1}+\left(U^{-1}+T^{\left(\sigma_{1}-1\right) / 2+\varepsilon}\right) \log M+M^{2} T^{\sigma_{1}-1+\varepsilon} U^{1+\varepsilon}\right) .
$$

Theorem 1 follows by setting $M=U=T^{\left(1-\sigma_{1}\right) / 4}$.

\section{LATTiCE POINTS IN GENERAL DOMAINS}

First we prove a technical lemma.

Lemma 8. Let $[a, b] \subset[0,1]$ and $\phi:[a, b] \rightarrow \mathbf{R}_{+}$be bounded. For $j=1, \ldots, M$ set

$$
I_{j}=[a+(j-1)(b-a) / M, a+j(b-a) / M] .
$$

Then there exists a constant $C$ depending at most on $\Gamma, z$ and $\varepsilon$ such that

$$
\begin{aligned}
& \frac{b-a}{M \operatorname{Vol}(\Gamma \backslash \mathbf{H})} \sum_{j=1}^{M} 1 /\left(\sup _{\omega \in I_{j}} \phi(\omega)\right)-C M(1 / \inf \phi)^{\left(3+\sigma_{1}\right) / 4+\varepsilon} \leq \mathcal{N}_{\Gamma}\left(G_{\phi} ; z\right) \\
& \leq \frac{b-a}{M \operatorname{Vol}(\Gamma \backslash \mathbf{H})} \sum_{j=1}^{M} 1 /\left(\inf _{\omega \in I_{j}} \phi(\omega)\right)+C M(1 / \inf \phi)^{\left(3+\sigma_{1}\right) / 4+\varepsilon}
\end{aligned}
$$

for any $M \geq 1$.

Proof. Define

$$
G_{\phi}^{j}=\left\{z \in G_{\phi}: \operatorname{Re}(z) \in I_{j}\right\} .
$$

By Theorem 1 we see that

$$
\mathcal{N}_{\Gamma}\left(G_{\phi} ; z\right)=\sum_{j=1}^{M} \mathcal{N}_{\Gamma}\left(G_{\phi}^{j} ; z\right)+O\left(M(1 / \inf \phi)^{\left(3+\sigma_{1}\right) / 4+\varepsilon}\right) .
$$


The error term comes from counting the end points twice. Applying Theorem 1 we get the estimate

$$
\begin{aligned}
& \frac{b-a}{M\left(\sup _{\omega \in I_{j}} \phi(\omega)\right) \operatorname{Vol}(\Gamma \backslash \mathbf{H})}-C^{\prime}(1 / \inf \phi)^{\left(3+\sigma_{1}\right) / 4+\varepsilon} \leq \mathcal{N}_{\Gamma}\left(G_{\phi}^{j} ; z\right) \\
& \leq \frac{b-a}{M\left(\inf _{\omega \in I_{j}} \phi(\omega)\right) \operatorname{Vol}(\Gamma \backslash \mathbf{H})}+C^{\prime}(1 / \inf \phi)^{\left(3+\sigma_{1}\right) / 4+\varepsilon} .
\end{aligned}
$$

The result now follows by summing over $j=1, \ldots, M$.

Our first application of the lemma is to prove Theorem 2.

Proof of Theorem 2. Set

$$
\begin{aligned}
\phi_{j}^{-} & =\sup _{\omega \in I_{j}} \phi(\omega), \\
\phi_{j}^{+} & =\inf _{\omega \in I_{j}} \phi(\omega) .
\end{aligned}
$$

Using the fact that $\phi$ (and hence $1 / \phi$ ) is monotonic we see that

$$
\begin{aligned}
\left|\int_{a}^{b} \phi(x)^{-1} d x-\frac{b-a}{M} \sum_{j=1}^{M} \phi_{j}^{ \pm-1}\right| & =\left|\sum_{j=1}^{M} \int_{I_{j}}\left(\phi(x)^{-1}-\phi_{j}^{ \pm-1}\right) d x\right| \\
& \leq((1 / \inf \phi)-(1 / \sup \phi)) / M .
\end{aligned}
$$

Thus, Lemma 8 implies that

$$
\left|\mathcal{N}_{\Gamma}\left(G_{\phi} ; z\right)-\frac{\operatorname{Vol}\left(G_{\phi}\right)}{\operatorname{Vol}(\Gamma \backslash \mathbf{H})}\right| \leq \frac{(1 / \inf \phi)}{M \operatorname{Vol}(\Gamma \backslash \mathbf{H})}+C M((1 / \inf \phi))^{\left(3+\sigma_{1}\right) / 4+\varepsilon} .
$$

The result now follows by choosing $M=\left[(1 / \inf \phi)^{\left(1-\sigma_{1}\right) / 8}\right]$.

We can now prove Proposition 5

Proof of Proposition 5. We will assume that $F$ is increasing (the case where $F$ is decreasing can be treated in a similar fashion). Let $a=\min (F)$ and $b=\max (F)$ and define $f_{+}, f_{-}:[a, b] \rightarrow \mathbf{R}_{+}$by

$$
\begin{aligned}
& f_{+}(t)=\inf F^{-1}(t), \\
& f_{-}(t)=\sup F^{-1}(t)
\end{aligned}
$$

for $t \in[a, b]$. The functions $f_{+}$and $f_{-}$are both well defined (by the intermediate value theorem) and they are both monotonic (increasing). Define

$$
\begin{aligned}
& \phi_{+, T}(t)=f_{+}(t) / T, \\
& \phi_{-, T}(t)=f_{-}(t) / T
\end{aligned}
$$

and

$$
\begin{aligned}
& \Omega_{-, T}=\left\{x+i y \in \mathbf{H}: y \geq \beta T^{-1}, x \in I\right\}, \\
& \Omega_{+, T}=\left\{x+i y \in \mathbf{H}: y>\beta T^{-1}, x \in I\right\} .
\end{aligned}
$$

We see that

$$
G_{\phi_{-, T}}-\Omega_{-, T} \subset \Omega_{F, T} \subset G_{\phi_{+, T}}-\Omega_{+, T}
$$

and

$$
\left(G_{\phi_{+, T}}-\Omega_{+, T}\right)-\left(G_{\phi_{-, T}}-\Omega_{-, T}\right)=\left(G_{\phi_{+, T}}-G_{\phi_{-, T}}\right) \cup\left(\Omega_{-, T}-\Omega_{+, T}\right) .
$$


By Theorem 1 we get

$$
\mathcal{N}_{\Gamma}\left(\left(\Omega_{-, T}-\Omega_{+, T}\right) ; z\right)=O\left(T^{\left(3+\sigma_{1}\right) / 4+\varepsilon}\right) .
$$

Corollary 3 implies that

$$
\mathcal{N}_{\Gamma}\left(G_{\phi_{+, T}}-G_{\phi_{-, T}} ; z\right)=\frac{\operatorname{Vol}\left(G_{\phi_{+, T}}-G_{\phi_{-, T}}\right)}{\operatorname{Vol}(\Gamma \backslash \mathbf{H})}+O\left(T^{\left(7+\sigma_{1}\right) / 8+\varepsilon}\right)
$$

and

$$
\begin{aligned}
\operatorname{Vol}\left(G_{\phi_{+}, T}-G_{\phi_{-, T}}\right) / T \\
\quad=\int_{a}^{b}\left(f_{+}(x)^{-1}-f_{-}(x)^{-1}\right) d x \\
\quad \leq \frac{b-a}{n} \sum_{j=1}^{n}\left(f_{+}((j-1)(b-a) / n+a)^{-1}-f_{-}(j(b-a) / n+a)^{-1}\right) \\
\quad \leq \frac{2(b-a)\left(\alpha^{-1}-\beta^{-1}\right)}{n} .
\end{aligned}
$$

Since this holds for all $n \geq 1$ we conclude that

$$
\operatorname{Vol}\left(G_{\phi_{+, T}}-G_{\phi_{-, T}}\right)=0
$$

for all $T$. Thus it follows that

$$
\mathcal{N}_{\Gamma}\left(\Omega_{F, T} ; z\right)=\frac{\operatorname{Vol}\left(\Omega_{F, T}\right)}{\operatorname{Vol}(\Gamma \backslash \mathbf{H})}+O\left(T^{\left(7+\sigma_{1}\right) / 8+\varepsilon}\right),
$$

and since

$$
\operatorname{Vol}\left(\Omega_{F, T}\right)=\int_{\alpha / T}^{\beta / T} \int_{\min (F)}^{F(T y)} \frac{d x d y}{y^{2}}=T \int_{\beta^{-1}}^{\alpha^{-1}}(F(1 / t)-\min (F)) d t,
$$

the proposition follows.

\section{ACKNowledgements}

The author would like to thank M. Risager for interesting discussions on the subject. The author would also like to thank A. Gorodnik and the anonymous referee for their comments.

\section{REFERENCES}

[1] F. Chamizo. Hyperbolic lattice point problems. Proc. Amer. Math. Soc. 139 (2011), no. 2, 451-459. MR2736328(2011m:11203)

[2] J. Delsarte. Sur le gitter fuchsien. C. R. Acad. Sci. Paris 214 (1942), 147-179. MR0007769 $(4: 191 \mathrm{a})$

[3] P. Erdös and P. Turán. On a problem in the theory of uniform distribution. I. Indag. Math. 10 (1948), 370-378. MR0027895(10:372c)

[4] A. Good. Local analysis of Selberg's trace formula. Lecture Notes in Mathematics 1040. Springer-Verlag, Berlin (1983). MR727476 (85k:11026)

[5] H. Huber. Zur analytischen Theorie hyperbolischen Raumformen und Bewegungsgruppen. Math. Ann. 138 (1959), 1-26. MR0109212 (22:99)

[6] H. Huber. Zur analytischen Theorie hyperbolischer Raumformen und Bewegungsgruppen. II. Math. Ann. 142 (1960/1961), 385-398. MR0126549(23:A3845)

[7] H. Huber. Zur analytischen Theorie hyperbolischer Raumformen und Bewegungsgruppen. II nachtrag. Math. Ann. 143 (1961), 463-464. MR0154980(27:4923)

[8] A. Gorodnik and A. Nevo. Counting lattice points. J. Reine Angew. Math. 663 (2012), 127176. 
[9] H. Iwaniec. Spectral methods of automorphic forms. Second edition. Graduate Studies in Mathematics 53. Amer. Math. Soc., Providence, RI (2002). MR1942691 (2003k:11085)

[10] S. J. Patterson. A lattice-point problem in hyperbolic space. Mathematika 22 (1975), 81-88. MR0422160(54:10152)

[11] M. Risager and Z. Rudnick. On the statistics of the minimal solution of a linear Diophantine equation and uniform distribution of the real part of orbits in hyperbolic spaces. Spectral analysis in geometry and number theory, 187-194, Contemp. Math. 484. Amer. Math. Soc., Providence, RI (2009). MR1500148(2010b:11076)

[12] M. Risager and J. Truelsen. Distribution of angles in hyperbolic lattices. Q. J. Math. 61 (2010), no. 1, 117-133. MR2592028(2011i:11102)

Department of Mathematical Sciences, Aarhus University, Ny Munkegade 118, DK8000 Aarhus C, Denmark

E-mail address: lee@imf.au.dk 